\title{
ARTICLES
}

\section{BASIC CARDIOPULMONARY LIFE SUPPORT (BCLS) - A REVIEW}

Mr. Shridhar Pujari. * | Dr. Savithri K. **

* Ph.D. scholar at Himalayan University at Itanagar, Arunachal Pradesh, India.

** Research Guide, Himalayan University at Itanagar, Arunachal Pradesh, India. DOI: http://doi.org/10.47211/trr.2019.v05i02.008

Received $25^{\text {th }}$ October 2019, Accepted $6^{\text {th }}$ December 2019, Published $20^{\text {th }}$ December 2019

\section{ABSTRACT}

$B C L S$ refers to the recognition of sudden cardiac arrest, calling for help, supporting the circulation, maintaining the airway and supporting breathing without the use of equipment other than personal protective devices. This skill could be used by a layperson or a healthcare provider in both out-of-hospital and in-hospital settings. BCLS is a level of medical care used in emergency, life-threatening situations until the person gets professional care by a team of paramedics or at a hospital. It can help of great help to people who are choking, drowning or are suffering from heart attack, and it can save life of some people. Review of related literature was done by a very careful perusal of researches already done and write ups published or lectures delivered on issues directly or indirectly connected with Basic Cardiopulmonary Life Support (BCLS). The present review article helped to see other people's views and perspectives regarding BCLS. It helped in identifying recent and significant advances in this area.

Key word: Basic Cardiopulmonary Life Support (BCLS), Literature review cardiac arrest.

\section{About Authors:}

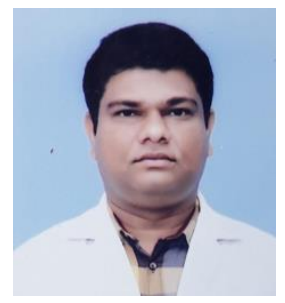

Author Mr. Shridhar Pujari is a Ph.D. scholar at Himalayan University at Itanagar in the Indian state of Arunachal Pradesh.

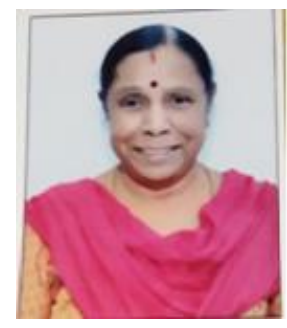

Author Dr. K. Savithri, M.Sc (N), PhD (N), is Research Guide at Himalayan University at Itanagar in the Indian state of Arunachal Pradesh. She has published papers in national and International Journals. She has attended and organised various National and International conferences and has given extensive lectures. 


\section{ARTICLES}

\section{INTRODUCTION}

Cardiovascular diseases are the major public health concern worldwide. Cardiopulmonary arrest is an emergency and requires immediate action with appropriate cardiopulmonary resuscitation (CPR) for an optimal outcome. Majority of cardiac arrests occur outside the hospital and timely management is essential. The outcomes, especially neurological, are poor in victims of cardiac arrest outside the hospital. Immediate cardiac compression by bystander can help buy time till medical help reaches and can improve the outcomes. The investigator made a thorough search of research studies, reports and publications brought out in various department of nursing field in India and abroad. The computer search was also done for collecting the literature related to the present study.

\section{Literatures associated to the prevalence on cardiac arrest.}

2. Literatures associated to knowledge and skills on BCLS.

3. Literatures associated to effectiveness of BCLS educational training module.

\section{Literatures associated to the prevalence on cardiac arrest}

(J Clin Med et.al, 2019) A study was conducted on sudden cardiac cessation and deaths of sportsman are dramatic and psychologically disturbing conditions for health care providers, family, and community. Strong physical exercise can stimulate the cardiac cessation and sometime sudden death may occur due to the known or unknown cardiac disorders and risk factors. The present formal assessment found that unexpected death related to cardiac cessation was not a common situation in sportsman. This includes the action of health care workers and common peoples in basic life support, finding and assessment before-participation in sports events and health information for promotion of the early identification of cardiac problems and early action of BLS performed during a short interval of time to automatic external defibrillator to better patient survival.

(Garg, Rakesh et al 2017). The cardiopulmonary resuscitation (CPR) guideline of comprehensive cardiopulmonary life support (CCLS) for management of the patient with cardiopulmonary arrest in adults provides an algorithmic step-wise approach for optimal outcome of the patient inside the hospital by trained medics and paramedics. This guideline has been developed considering the infrastructure of healthcare delivery system in India. This is based on evidence in the international and national literature. In the absence of data from the Indian population, the extrapolation has been made from international data, discussed with Indian experts and modified accordingly to ensure their applicability in India. The CCLS guideline emphasise the need to recognise patients at risk for cardiac arrest and their timely management before a cardiac arrest occurs. The basic components of CPR include chest compressions for blood circulation; airway maintenance to ensure airway patency; lung ventilation to enable oxygenation and defibrillation to convert a pathologic 'shockable' cardiac rhythm to one capable to maintaining effective blood circulation. CCLS emphasises incorporation of airway management, drugs, and identification of the cause of arrest and its correction, while chest compression and ventilation are ongoing. It also emphasises the value of organised team approach and optimal post-resuscitation care. 


\section{ARTICLES}

(Anilkumar 2010). A study was carried out by research team, Sent, Jon's health sciences in Bangalore; data was collected from 21000 coronary patients from 89 hospitals across at 50 cities across country. They found that out of 20,468 patients who have given definitive diagnosis, $60 \%$ showed evidence of heart attack, compared to $40 \%$ in developed countries. By 2020, India will have the largest population suffering from Coronary artery disease, India will consider one $3^{\text {rd }}$ of death due to Heart disease and about 20,000 clients may suffer with heart problems every day in India. About 60 million Indians unpleasant from Heart disease and $30 \%$ more are at a high risk.

According WHO cardiovascular disorders are the number one reason for death globally; WHO census of September 2016 shows, 17.9 million humans die every year from cardiovascular diseases, an approximate $31 \%$ of whole deaths in worldwide. Among those deaths, an approximate 7.4 million are related to coronary artery disorders and 6.7 million are because of stroke, $85 \%$ of all cardiovascular disease deaths are due to heart attacks and strokes.

\section{Literatures associated to the knowledge and skill of BCLS.}

(Abolfotouh MA et al. 2018) A study was done to assess the impact of basic care life support training education and attitudes of health care professionals to begin CPR and utilization of automated external defibrillator to identify the fact which affect these attitudes. Study included 321health-care providers who were trained a BLS-AED course as post-BLS group, 421 selected as pre-BLS group who were not trained. Questionnaire was given to both groups to assess the status of basic care life support education. Result shows that $53.4 \%$ of pre-BLS shows positive attitudes, and it was $64.8 \%$ in post-BLS groups. Continuing of BLS education can improve knowledge and skill about CPR practice and utilization of AED.

Min, Byoung et al (2004). A cardiopulmonary life support system is disclosed. The life support system comprises a housing defined by a top side, a bottom side, and an inner periphery. First and second tubes are adjacent to each other in the housing, and the first and second tubes each have an input port and an output port. An alternating member is attached to the housing and disposed between the first and second tubes. The alternating member alternately squeezes the first and second tubes.

\section{Literatures associated to effectiveness of educational training module.}

(Beom, Jin \& Kim, Ji \& Chung, Hyun \& Kim, Su \& Ko, Dong \& Cho, Junho. 2018)The effects of the flipped classroom have been demonstrated in various fields of education in recent years. Training in emergency medicine is also beginning to gradually implement the flipped classroom; however, its practical effect in emergency medicine contexts is not yet clear. The present study investigates the effects of the flipped classroom on advanced cardiopulmonary life support (ACLS) training implemented among practicum students in emergency medicine. The study randomly assigned into control and experimental conditions 108 fourth year students in the College of Medicine at Yonsei University, in Seoul, who were scheduled to take clinical practice in emergency medicine between March and July 2017. Students were taught about ACLS in either a traditional lecture-based classroom (control condition) or a flipped classroom (experimental condition); then, simulation training with ACLS scenarios was carried out. Finally, each student was rated on performance using a rating form developed in advance. ACLS simulation scores of the students in the flipped classroom were $70.9 \pm 10.9$, which was higher than those of the 


\section{ARTICLES}

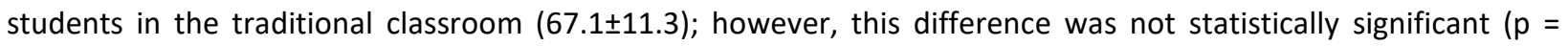
0.339). In addition, the difference in student satisfaction as measured on a survey was statistically insignificant ( $p=$ 0.655). Competency assessment after simulation-based training in ACLS undergone by senior medical students randomly assigned to flipped and traditional classrooms showed no statistical difference in competency between the two groups.

(Jung, Hyo-Ju \& Chae, Min-Jeong. 2015). This study is a quasi-experiment with a pre-post design and a nonequivalent control group performed from May 1 to June 2, 2013 to verify the effects of a simulation based education program on nursing students' self-confidence, and clinical performance ability. Methods: The subjects of the study were a total of 60 students in their fourth year of a nursing program at a university located in $\mathrm{G}$ city and they were assigned to an experimental group of 30 students and a control group of 30 students by convenience sampling. Results: After being offered education, self-confidence, and clinical performance ability were significantly more improved than before in each group. In the comparison of the two groups, the self-confidence $(t=3.00, p=.004)$ and clinical performance ability $(t=3.14, p=.003)$ of the experiment group were significantly higher than those of the control group. Conclusions : The results indicated that instructional methods using a simulation-based emergency care program should be adopted maximize the effects of advanced cardiopulmonary life support education.

Basic Care Life Support (BCLS) is a level of medical care used in emergency, life-threatening situations until the victim can be cared for professionally by a team of paramedics or at a hospital. It can help people who are choking, drowning or are suffering from cardiac arrest, and it can make the difference between life and death for some people. Hence the importance of BCLS knowledge and skills in order to deal with emergencies combined with, the nursing officers with their clinical expertise, is very important. It's important for them to know same basic skills in BCLS.

(Nirmala. K. 2013) A study was conducted to assess the success of educational programme intern's awareness and practice on cardiopulmonary resuscitation among 40 nursing officers in Punjab, by using purposive convenient sample. The investigator assessed the knowledge on CPR and practice on CPR, before and after administering a teaching module Data analyzed used by descriptive and inferential statistics, results of study revealed that post test knowledge score (36.75) was significantly higher than the pre-test (28.53). Study concluded that pre test awareness and practice on cardiopulmonary resuscitation among nursing officers was not satisfactory, but there was a significant increase in level of knowledge and practice of CPR after the administration of teaching module.

\section{CONCLUSION}

The need for BCLS knowledge and skills in order to deal with emergencies combined with, the nursing officers with their clinical expertise, was urgently felt. Nursing officers play vital role in building strong nursing care providers. They act as a role model and carrying the leadership required to introduce witness based practice. Hence, urgency to do practical research and also to train the health care professionals about knowledge and skills regarding basic care life support was felt. 


\section{ARTICLES}

\section{REFERENCES}

1. Abolfotouh MA, Alnasser MA, Berhanu AN, Al-Turaif D, Alfayez Al, King Abdullah International Medical Research Center, King Saud bin-Abdulaziz Universit DOI: 10.1186/s12913-017-2621-5, PMC5610457PMID: 28938914 Indexed for MEDLINE 2018

1. Alvárez, A.C. \& López-Herce, Jesús \& Torrero, R.M. \& Pérez, L.S. (1999). Teaching pediatric basic life support to medical students in Spain. Current Paediatric Research. 3. 13-19.

2. Beom, Jin \& Kim, Ji \& Chung, Hyun \& Kim, Su \& Ko, Dong \& Cho, Junho. (2018). Flipped-classroom training in advanced cardiopulmonary life support. PLOS ONE. 13. e0203114. 10.1371/journal.pone.0203114.

3. Camacho, Hernando. (2011). Advanced cardiopulmonary life support (Second Part): Changes to be made for advanced cardiopulmonary life support according to the 2010 guidelines presented in Chicago. Revista Colombiana de Cardiología. 18. 65-79.

4. Garg, Rakesh \& Ahmed, SyedMoied \& Kapoor, Mukul \& Rao, SSCChakra \& Mishra, BibhutiBhusan \& Kalandoor, MVenkatagiri \& Singh, Baljit \& Divatia, Jigeeshu. (2017). Comprehensive cardiopulmonary life support (CCLS) for cardiopulmonary resuscitation by trained paramedics and medics inside the hospital. Indian Journal of Anaesthesia. 61. 883. 10.4103/ija.IJA_664_17.

5. J Clin Med et.al, 2019 Apr 24; 8(4).pii: E556. doi: 10.3390/jcm8040556.Prevention of Sudden Death Related to Sport: The Science of Basic Life Support-from Theory to Practice.Center for Physical Education \& Sports, Federal University of EspíritoSanto, Vitória 29075810, Brazil.

6. Jung, Hyo-Ju \& Chae, Min-Jeong. (2015). Effect of Simulation-based Advanced Cardiopulmonary Life Support Education for Nursing Students Hospitals. The Korean Journal of Health Service Management. 9. 127-143. 10.12811/kshsm.2015.9.3.127.

7. Min, Byoung \& Won, Yu Sam \& Sun, Kyung \& Baek, Kwang \& Lee, Hyuk \& Roh, Yang \& Hwang, Chang \& Lee, Whang \& Lee, Jung Chan. (2004). Cardiopulmonary life support system.

8. Nirmala.K. Effectiveness of a planned teaching module on knowledge \& practice of cardiopulmonary resuscitation among nursing officer. Nursing officer of India 2013 Feb;7(2):4-6.

9. Smeltzer C, Bare G B. Brunner and suddarth's (2004) text book of Medical surgical Nursing. $10^{\text {th }}$ ed. Philadelphia, Lippincott Williams and Wilkins .P:233. 